\title{
Thermal structure of solid flames
}

\author{
A. A. Zenin \\ Institute of Chemical Physics, USSR Academy of Sciences, \\ 117334 , Moscow, USSR
}

\begin{abstract}
A brief outline of thermal structure investigations of self-propagating high-temperature synthesis (SHS) of metalboron, metal-silicon and metal-carbon is given. Multi-stages (multi-zones) of synthesis wave are revealed. Relative reaction zone sizes (wide or narrow) are defined. Positions in the wave of the stages governing the propagation rate are found. A chemical scheme of titanium silicide synthesis is suggested. Profiles of the volumetric heat release rate in the wave are obtained from experimental temperature profiles which permits to establish formal kinetic laws. Interpretations of these laws are proposed.
\end{abstract}

\section{INTRODUCTION}

The method of self-propagation high-temperature synthesis (SHS) permits to obtain materials with predicted properties (ref. 1) provided that detailed knowledge of synthesis processes is available. It makes it necessary to investigate various characteristics of synthesis waves. The most important of these characteristics are temperature wave profiles. Among other methods of temperature measurement in combustion waves the thermocouple method proved to be the most informative one. It was substantiated (refs. 2,3 ). Errors of the thermocouple measurements in the synthesis waves are very small and can be neglected if the thickness of thermocouple $h$ and the length $P$ of the isothermal part of the thermocouple (parallel to the isotherms of the synthesis waves) meet the following requirements:

$$
\begin{aligned}
& h \approx 0,2 \mathrm{a} / \mathrm{r}_{6} \\
& l \geqslant 100 \mathrm{~h}
\end{aligned}
$$

Here: a - the thermal diffusivity of the medium, $z_{b}$ - linear burning rate. There exist different ways of protecting thermocouples from interaction with the medium. Tungsten-rhenium thermocouples used by us have very slight interaction (refs. 3,4). Fig. I shows the position of thermocouple embedded in the sample which is to be ignited at the top.

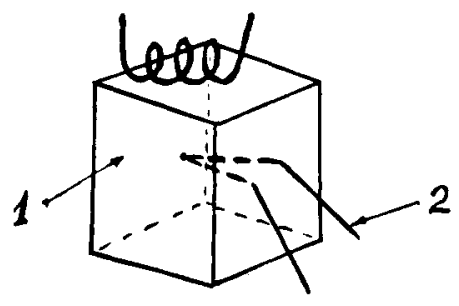

Fig. 1. Embedding of thermocouple

(2) in the sample (1).

Samples are burned in argon at pressures $1-20 \mathrm{~atm}$. The object of this article is to show how the characteristics of the thermal structure wave should be used to obtain the physics and chemistry of the synthesis process. 


\section{TEMPERATURE PROFILES AND PHYSICS OF COMBUSTION}

The temperature field in sHs wave as shown in ref. 5 is monodimensional since the diffusion processes in the solid are much slower than the heat transfer. The results obtained point to a great variety of temperature profile types in SHS wave. As a rule, the process of synthesis consists of several stages (zones). The synthesis waves of monoborides have two stages (refs. 3,6), see Fig. 2.

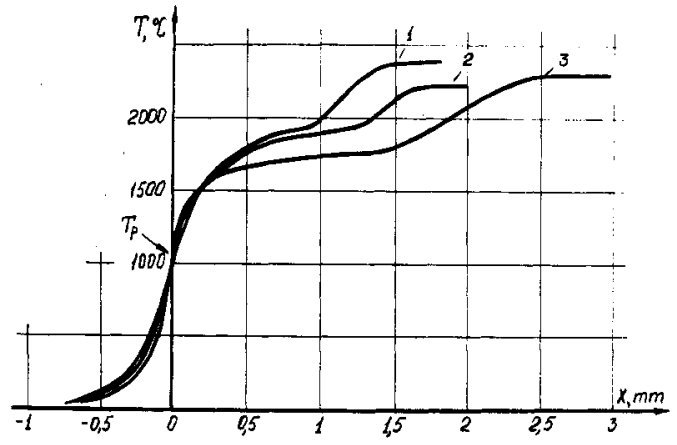

Fig. 2. Averaged temperature profiles of SHs waves. Synthesis of borides.

1. Nb-fine+B;

2. $\mathrm{Nb}+\mathrm{B} ; 3$. $\mathrm{Ta}+\mathrm{B}$.

Here the first stage is the synthesis of diborides which decompose later. The synthesis waves of titanium carbide can have two or three stages (ref. 4), see Fig. 3.

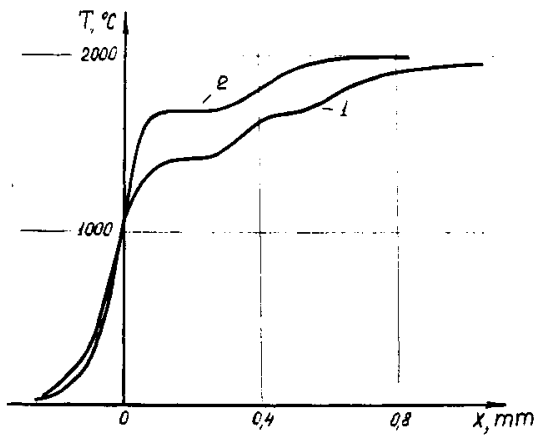

Fig. 3. Averaged temperature profiles of SHS waves. System $\mathrm{Ti}+0.5 \mathrm{C}$ (soot $\mathrm{TG}-$ 10). Variation of Ti particle sizes.

1. $-32+25 \mathrm{~m} \mathrm{~m}$;

2. $-63+50 \mathrm{~mm}$.

The synthesis wave of titanium silicide can have four stages (refs. 3,7), see Fig. 4 (for the processes in these stages - see below).
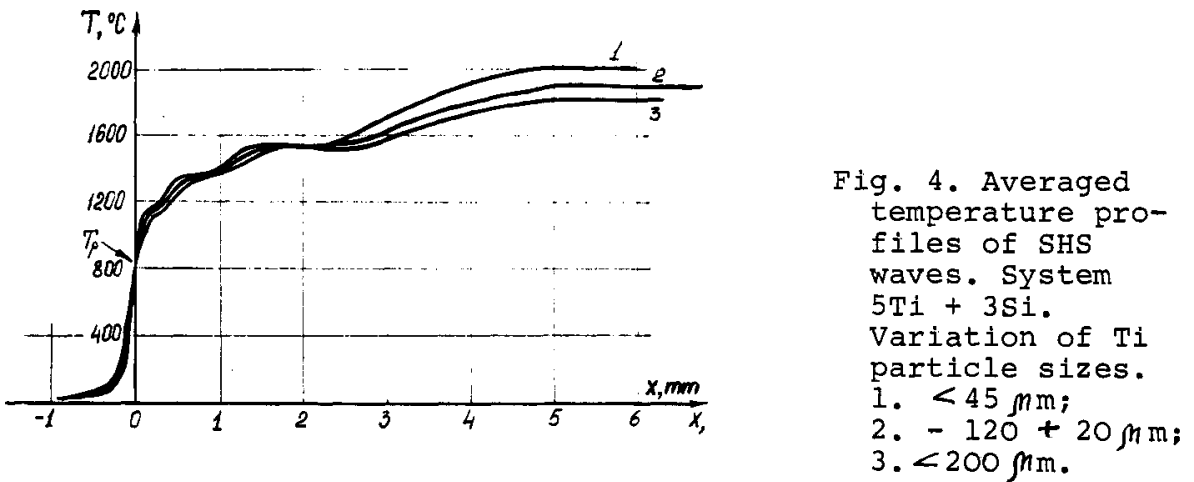

The relative zone size $\Delta$ is equal to the real zone size $I$ (i.e. the region of zone that is characterised by a quick change of temperature; usually I is a distance from the initial temperature of zone to the 0.9 final temperature of this zone) divided by a conductive size $I_{m}$ of this zone $\left(\mathrm{Lm}_{\mathrm{m}}=\mathrm{a} / \mathrm{z}_{6}\right.$, where a - the coefficient of the thermal diffusivity of the zone medium). Thus, $\Delta=L / L_{m}$. By definition a zone is narrow if its relative size $\Delta \simeq 1$ 
and it is wide if $\Delta \gg 1$. In sHs waves there are zones of both types. Single zone profiles always have a wide reaction zone - see meaning of $\Delta$ in Table 1 for systems SHS with single zone.

TABLE 1. Relative zone sizes $\Delta$ and $\Delta_{1}$.

\begin{tabular}{|c|c|c|c|c|c|c|c|c|c|}
\hline No & System & $z_{6}, \frac{\mathrm{cm}}{\mathrm{s}}$ & $\Delta$ & $\Delta_{1}$ & No & System & $\tau_{b}, \frac{\mathrm{cm}}{\mathrm{s}}$ & $\Delta$ & $\Delta_{1}$ \\
\hline 1 & $\mathrm{Nb}+\mathrm{B}$ & 0.55 & & 4.5 & 6 & $\mathrm{Zr}+\mathrm{Si}$ & 1.5 & 40 & \\
\hline 2 & $\mathrm{Nb}+2 \mathrm{~B}$ & 0.62 & 3.5 & & 7 & $\mathrm{Ti}+\mathrm{Si}$ & $0.5-1.5$ & & $0.7-1.1$ \\
\hline 3 & $\mathrm{Ta}+2 \mathrm{~B}$ & 0.63 & 12 & & 8 & $\mathrm{Ti}+\mathrm{C}$ & 7.0 & & 2.9 \\
\hline 4 & $\mathrm{Zr}+2 \mathrm{~B}+308 \mathrm{ZrB}_{2}$ & 1.8 & 13.5 & & 9 & $\mathrm{Ti}+0.8 \mathrm{C}$ & 3.5 & & 4.5 \\
\hline 5 & $\mathrm{Hf}+2 \mathrm{~B}$ & 2.2 & 18 & & 10 & $\mathrm{Ti}+0.5 \mathrm{C}$ & 1.2 & & 2.1 \\
\hline
\end{tabular}

Profiles with two or three zones often have a wide first reaction zone (see meanings of $\Delta_{1}$ in Table $1 ; \Delta_{1}$ - is the relative size of the first reaction zone of multi-zone sHS systems), the only exception being multi-zone profiles of synthesis wave of titanium silicide (see system 7 in Table 1 ) consisting of narrow zones divided by isothermic sections. The establishment of relative zone sizes facilitates to spot the region of the wave governing the burning rate. As was shown in refs. 2,8 , the following equation is valid for wide reaction zones:

$$
z_{B}=k \int_{0}^{\infty} \Phi(x) \exp \left(-x / L_{m}\right) d x
$$

where: $\quad k$ - coefficient, $\Phi(x)$-distribution of heat release rate in synthesis wave, $\boldsymbol{x}$ - co-ordinate that has 0 at the beginning of the reaction zone at the temperature $T_{p}$. It can be seen from eq. (1) that the burning rate in the wide reaction zone is mostly affected by the low-temperature portion of this zone. Indeed, at $x>L_{m}$ the contribution of $\Phi(x)$ to the integral and, consequently, the effect on $z_{B}$ drops sharply because of the multiplier exp $\left(-x / L_{m}\right)$. In contrast, in narrow zones the propagation rate is determined by the zone maximum temperature. However, in investigated system 7 of this type (see Table 11 the maximum temperature of the first zone is equal to $\sim 1150^{\circ} \mathrm{C}$. Thus, the propagation rate in the system under consideration is controlled by its low-temperature portion. From what was said above it follows that the experimental dependences of the initial system parameters are affected by changes in the controlling stage.

Another problem of physics of burning is concerned with the interaction of synthesis wave zones (refs. $4,6,7,8$ ). In ref. 9 three different regimes of zone interaction are established: joining zone regime, governing zone regime and tearing off zone regime. Single-stage temperature profile indicates, as a rule, the joining zones' regime (if the system parameters are being varied multi-stage zone occur). The governing zone regime can be observed in the system $\mathrm{Ti}-\mathrm{Si}$, when the high-temperature stage with the final temperature $\sim 1150^{\circ} \mathrm{C}$ (see below) imposes its propagation rate on the low-temperature stage at $880^{\circ} \mathrm{C}$ corresponding to the transfer $\mathrm{Ti}_{\alpha} \rightarrow \mathrm{Ti}_{\beta}$. There are many cases of tearing off zone regimes: these are almost all the cases of multi-stage structures of synthesis wave of titanium silicide - see Fig. 4. Note, the isothermical portions that separate stages in Fig. 4 do not indicate absence of reactions. These are either zones of thermo-neutral transformations or heatabsorption (for instance in melting processes), which is balanced by heat release in the same zone. This regime of burning is called stefans' regime.

\section{TEMPERATURE PROFILES AND SYNTHESIS MECHANISM}

An approximate scheme of the chemical processes in the synthesis wave can be composed on the basis of the wave temperature profiles, of the thermodynamic diagrams and in the data received from $x$-ray structural analysis of final products. It is important that a temperature profile has as many isothermal portions as possible. Their temperature values are used to identify intermediate compositions of a reagent. X-ray analysis of systems with ballast is 
also used for additional identification. The Identification permits to analyse both the final products and the intermediate ones. In the latter case systems having ballast are used.

Let us consider titanium silicide synthesis as an example (refs. 3,7). The study of its thermal structure zone shows that changes in the composition of systems, in particle sizes and other parameters of systems do not affect the temperature of the isothermal portions in the synthesis wave that are equal to $1150^{\circ} \mathrm{C}, 1350^{\circ} \mathrm{C}$ and $1530^{\circ} \mathrm{C}$. All the changes in the composition bring about sufficient change only in the forth zone of the synthesis wave. $A$ comparison of the temperatures of isothermal portions and the thermodynamic diagram of $\mathrm{Ti}$ - Si makes it easy to identify processes in the synthesis wave zones. In fact, at the end of the first stage the temperature is almost equal to that of peritectic reaction:

$$
\mathrm{Ti}_{5} \mathrm{Si}_{3}+\mathrm{Ti}=\mathrm{Ti}{ }_{3} \mathrm{Si} ;\left(1170^{\circ} \mathrm{C}\right)
$$

The compound $\mathrm{Ti}_{3} \mathrm{Si}$ is not stable and decomposes when the temperature rises. At the end of the second stage the temperature is almost equal to that of eutectic:

$$
\mathrm{Ti}_{5} \mathrm{Si}_{3}+\mathrm{Ti} ;\left(1340^{\circ} \mathrm{C}\right)
$$

At the end of the third stage the temperature is almost equal to that of the melting $\mathrm{TiSi}_{2}\left(1500-1540^{\circ} \mathrm{C}\right)$. The final products are formed only at $\mathrm{T}>1530^{\circ} \mathrm{C}$ in the forth zone. The following characteristic features of synthesis of titanium silicides in SHS wave can be inferred from the obtained results. 1) The compound $\mathrm{Ti}_{5} \mathrm{Si}_{3}$ is an important ingredient of the initial period of synthesis. A great amount of it is formed during the first stage at $\mathrm{T}<1150^{\circ} \mathrm{C}$ which can be seen from the fact of its participation in reactions at $1170^{\circ} \mathrm{C}$ and at $1350^{\circ} \mathrm{C}$. The functions of the volumetric heat release rate $\Phi(\mathrm{x})$ were obtained from the experimental temperature profiles $T(x)$ (see below), the functions of $\Phi(x)$ being the highest ones in the temperature interval $800-1170^{\circ} \mathrm{C}$. The high heat release rate is due to the absence of diffusion impediment at the initial stage of synthesis wave and the large heat release at the formation of $\mathrm{Ti}_{5} \mathrm{Si}_{3}$ as well. As the first stage determines the synthesis wave rate one can conclude that the reaction of partial formation of $\mathrm{Ti}_{5} \mathrm{Si}_{3}$ is the main factor determining the propagation rate of synthesis wave of titanium silicides.

2) The initial components of the system, Ti and Si, begin reacting at $\sim 800^{\circ} \mathrm{C}$ that is long before they start melting $\left(\mathrm{T}_{\text {melt }}=1650^{\circ} \mathrm{C}\right.$ and $1420^{\circ} \mathrm{C}$, respectively). The experimental curves $T(x)$ have no melt isothermal portions at the temperatures of the melting of the initial components. It seems to indicate that some part of the process of silicide formation occurs in the solid phase. The liquid $\mathrm{Ti}$ and $\mathrm{Si}$ that are formed later react quickly, that is in the regime of chemical transformation in which there are no isothermal portions of melting of $T i$ and $S i$ at the curves $T(x)$.

3) Irrespective of the composition of the initial mixture the compound TiSi, with the maximum quantity of $\mathrm{Si}$, is formed in the sHs wave. Its presence is' revealed when it melts at the end of the third stage at $1530^{\circ} \mathrm{C}$. TiSi ${ }_{2}$ is formed even when $\alpha$ in the system $T i+\alpha S i$ is small (for example, $\alpha=0.45$ ). R-ray structural analysis reveals the presence of $\mathrm{Tisi}{ }_{2}$ in the products of synthesis provided the reactions of the fourth stage are suppressed by ballast being introduced. In the fourth stage TiSi2 decomposes if $\alpha<2$.

The process of silicide forming in sHs wave can be shown in the following scheme (refs. 3,7 ).

1) $\mathrm{Ti}_{\alpha} \rightarrow \mathrm{Ti}_{\beta} \quad\left(880^{\circ} \mathrm{C}\right)$

Zone 2 4) $\mathrm{Ti}_{5} \mathrm{Si}_{3}+\mathrm{Ti} \longrightarrow$ (eutectic, $1350^{\circ} \mathrm{C}$ )

Zone 3 5) $\mathrm{Ti}_{5} \mathrm{Si}_{3}, \mathrm{Ti}, \mathrm{Si} \longrightarrow \mathrm{TiSi}_{2}$, TiSi

6) melting $\mathrm{TiSi}_{2}\left(1530^{\circ} \mathrm{C}\right)$ 
In conclusion, I would like to note that the formation of compounds of metalnon-metal, with the second component having the maximum quantity in the intermediate products, is a typical phenomenon of sHs wave. It was also observed in sHS wave of borides (see above) and of titanium nitride (see refs. 10,11). The phenomenon can be accounted by the existence of a limiting stage which is a diffusion of non-metal through the layer of the halfproduct on the surface of metal particles. Indeed a quick transfer of non-metal to the surface of a metal particle leads to the saturation of the surface with nonmetal. The phenomenon becomes more distinct when metal melts in the reaction zone of SHS wave which increases the average size of particles. Further on the process of equalizing non-metal concentration along the radius of a particle causes decomposition of the compound with a maximum quantity of nonmetal when $\alpha$ is small.

\section{TEMPERATURE PROFILES AND ZONE MACROKINETICS}

Three types of curves are needed for establishing the macrokinetic characteristics of zones of SHS wave: the temperature profiles $T(x)$, the function of volumetric heat release rate $\Phi(x)$ and the function of the degree of reaction completeness $\eta(x) \quad\left(\eta=0\right.$ at $T=T_{P}$ and $\eta=1$ at the final temperature $\left.T_{f}\right)$. When $T(x)$ are experimentally obtained, the functions $\Phi(x)$ and $\eta(x)$ can be derived from the following system of equation:

$$
\begin{gathered}
d / d x[\lambda(x) d T / d x]-z_{b} \rho c d T / d x+\Phi(x)=0 \\
z_{b} \rho d \eta / d x+\Phi(x) / Q=0
\end{gathered}
$$

Here $\lambda, c$ and $\rho$ - thermal conductivity coefficient, specific heat capacity coefficient and density respectively. Typical examples of values of the parameters $\lambda$ and $c \rho$ are shown in Table 2 (index 1 corresponds to the initial mixtures, index 2 - to the final products). Table 2 shows that, as a rule, $c \rho$ changes slowly while $\lambda$ becomes several times higher.

TABLE 2. Thermal conductivity coefficients $\lambda$ and volumetric heat capacity coefficients $c \rho$ of initial mixtures (1) and of

\begin{tabular}{|c|c|c|c|c|c|}
\hline System & $\begin{array}{l}\lambda_{i} \cdot 10^{2}, \\
\mathrm{cal} / \mathrm{cm} \mathrm{s}^{\circ} \mathrm{C}\end{array}$ & $\begin{array}{c}c, \rho_{1} \\
\mathrm{cal} / \mathrm{cm}^{3}\end{array}$ & $\begin{array}{r}\lambda_{2} \mathrm{IO}^{2}, \\
\mathrm{O}_{\mathrm{C} \mathrm{cal} / \mathrm{cm} \mathrm{s}}\end{array}$ & $\begin{array}{c}c_{2} \rho_{2} \\
{ }^{\circ} \mathrm{C} \mathrm{cal} / \mathrm{cm}^{3}{ }^{\circ} \mathrm{C}\end{array}$ & $\begin{array}{c}\rho_{1}, \\
\operatorname{cal} / g r{ }^{\circ}\end{array}$ \\
\hline $\begin{array}{l}\mathrm{Nb}+\mathrm{B} \\
\mathrm{Nb}+2 \mathrm{~B} \\
\mathrm{Ta}+2 \mathrm{~B} \\
\mathrm{Zr}+2 \mathrm{~B} \\
\mathrm{Hf}+2 \mathrm{~B} \\
\mathrm{Ta}+\mathrm{B} \\
\mathrm{Zr}+\mathrm{Si} \\
5 \mathrm{~T} i+3 \mathrm{Si} \\
2 \mathrm{Zr}+\mathrm{Si}\end{array}$ & $\begin{array}{l}0.25 \\
0.48 \\
0.40 \\
0.97 \\
0.40 \\
0.28 \\
0.42 \\
0.47 \\
0.31\end{array}$ & $\begin{array}{l}0.45 \\
0.46 \\
0.46 \\
0.46 \\
0.48 \\
0.55 \\
0.22 \\
0.40 \\
0.22\end{array}$ & $\begin{array}{l}0.90 \\
1.2 \\
0.9 \\
2.0 \\
0.80 \\
0.50 \\
0.80 \\
0.80 \\
0.80\end{array}$ & $\begin{array}{l}0.54 \\
0.70 \\
0.6 \\
0.67 \\
0.50 \\
0.47 \\
0.22 \\
0.40 \\
0.22\end{array}$ & $\begin{array}{l}3.6 \\
3.0 \\
5.0 \\
3.0 \\
5.0 \\
6.0 \\
2.8 \\
2.0 \\
3.0\end{array}$ \\
\hline
\end{tabular}
products (2) of SHS wave.

To obtain the functions $\Phi(x)$ and $\eta(x)$ one has to know the dependence $\lambda(x)$ in the reaction zone. To be able to calculate the dependence $\lambda(x)$ it is necessary to assume a certain model of reaction. Let us assume that the reaction layer increases monotonously on metal particles and the geometry of the arrangement of particles remains constant during the reaction. This model is approximately valid for the synthesis wave of borides and silicides, in any case, this is true of the solid phase. The degree of the completeness of a reaction is defined by a portion of the substance that has reacted by a certain moment $\tau$. This portion can be expressed in terms of geometrical characteristics of media and the thermal conductivity of the three-phased 
system (metal, non-metal, product) that has been obtained can be calculated, provided the thermal conductivity of each of the phases is known (refs. 3,12). For the investigated systems $M e+\alpha B$, Me $+\alpha s i$ the obtained dependence can be described by the linear formula (true to $\sim 10 \%$ ):

$$
\lambda=\lambda_{2} \eta+\lambda_{1}(1-\eta)
$$

The accuracy of formula (4) does not depend on forms and sizes particles which means that this formula valid for reactions with the melting components. By solving the system of equation (2), (3), (4) we can obtain:

$$
\begin{gathered}
\eta=\frac{z_{B} \rho c\left(T-T_{0}\right)-\lambda_{1} d T / d x}{\left(\lambda_{2}-\lambda_{1}\right) d T / d x+z_{B} \rho Q} \\
\Phi=z_{B} \rho Q^{d \eta / d x}
\end{gathered}
$$

Here $Q$ the heat of the reaction, $T_{0}$ - initial temperature.

However, eq. (4) is not always valid. The investigation of carbide synthesis waves has revealed that the dependence $\lambda(x)$ is not monotonous. The function

$\lambda(x)$ for the synthesis waves $T i+\alpha C$ was determined experimentally by obtaining distribution of electroconductivity 3 in these waves. In refs. 13, 14 was shown that the electronic components of electroconductivity and thermal conductivity are much higher than the phonon conductivity for the carbide of Group 4 and Group 5 and for incomplete carbon materials (for soot for example). It follows that for the evaluation of $\lambda(x)$ in the carbide synthesis waves the measurements of electroconductivity $\boldsymbol{b}(x)$ in the same waves can be used. If the mechanism of heat-transfer and that of electro-transfer are identical, thermal conductivity $\lambda$ and electroconductivity $\boldsymbol{z}$ are interrelated in accordance with the law by Wiedemann-Franz:

$$
\lambda=6 L_{0} T
$$

Here I - coefficient. An additional electrode made of ribbon wire and embedded in the same horizontal section as the thermocouple was used in these experiments. The thermocouple acted as the second electrode. We set up a potential difference between the electrodes and recorded the voltage and the electric current. These experiments made it possible to determine the values of electroconductivity in the same places where the temperatures were measured. The experiments show that electroconductivity 3 . in the synthesis waves of titanium carbide decreases at the beginning of the reaction and increases then. The change of 3 is probably connected with the decrease of the density of soot between the metal particles at the beginning of the reaction which is due to diffusion of carbon atoms into a metal. The indicated interrelationship can be understood from the fact that electroconductivity of the initial sample is determined by the soot porous frame.

To obtain profiles $\Phi(x)$ and $\eta(x)$ according to eq. (2) and (3) the established dependencies $\lambda(x)$ in the synthesis waves of titanium carbide with different kinds of soot and with a different ratio of component were used. Thus three profiles: $T(x), \Phi(x)$ and $\eta(x)$ were obtained for each regime of boride-, silicide- and carbide synthesis. A typical example of these profiles is shown in Fig. 5 .

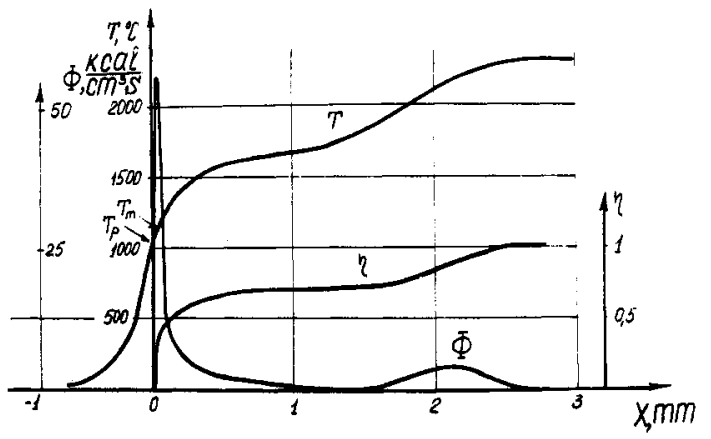

Fig. 5. Profiles $\mathrm{T}(\mathrm{x})$, $\Phi(x)$ and $\eta(x)$. system $\mathrm{Ta}+\mathrm{B}$. 
The profiles of these types for different rate values $\boldsymbol{z}_{6}$ for each system make it easy to find macrokinetic laws for each zone. Let us present $\Phi(\eta, T)$ in the form (ref. 15):

$$
\begin{gathered}
\Phi(\eta, T)=Q k_{0} \varphi(\eta) \exp (-E / R T) \\
\varphi(\eta)=\eta^{-n} \exp \left(-m_{0} \eta\right)
\end{gathered}
$$

Here: $Q K_{0}$ - coefficient, $\varphi(\eta)$ - kinetic law, $m_{0}$ - parameter of reaction deceleration in exponential law, $\boldsymbol{n}$ - power index. For obtaining activation energy $E$ the experimental values of $\Phi$ are plotted in coordinates $\lg \Phi$ v $1 / T$ the values of $\eta$ being constant. For obtaining kinetic law parameters the experimental data are plotted in coordinates $(\ln \Phi+E / A T)$ vs $\eta$ (or vs en $\eta$ ). If the data are placed on a straight line and the absciss is $\eta$; then $n$ is obtained, whereas when the absciss is $\ell_{n} \eta$, then $m_{0}$ is obtained. For an example of obtaining values $E, Q k_{0}$ and $n$ in the system $\mathrm{T} i+\alpha \mathrm{C}$ see Fig. $6(\mathrm{a}, \mathrm{b})$. Here the following equation was obtained:

$$
\Phi(\eta, T)=3 \cdot 10^{9} \eta^{-7} \exp (-55000 / R T), \mathrm{cal} / \mathrm{cm}^{3} \mathrm{~s}
$$
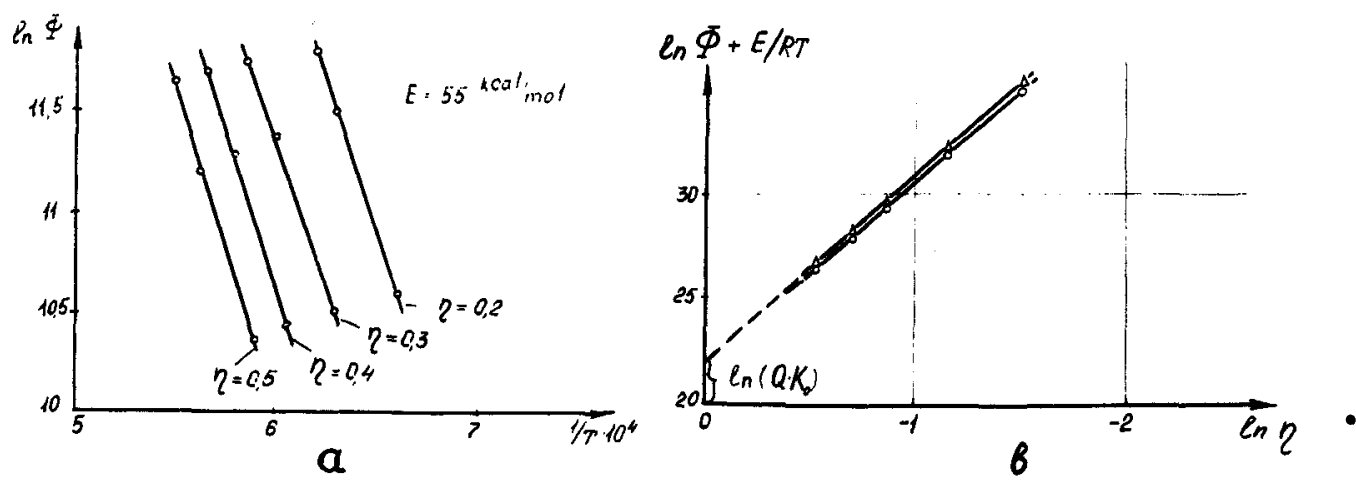

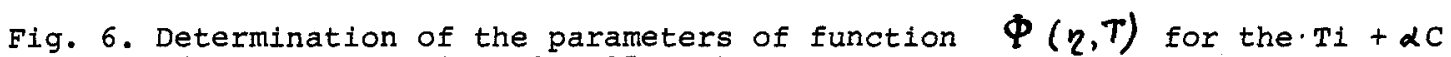
system (soot $\mathrm{Tg}-10$, Ti $-32+25 \mathrm{Mm}$ ).

a - determination of activation energy $E$.

$\mathrm{b}$ - determination of the parameters $n$ and $Q_{K}$.

The summing-up data of the macrokinetic characteristics of all the studied systems sHS are submitted in Table 3 .

TABLE 3. Macrokinetic characteristics of SHS system.

\begin{tabular}{lcccccc}
\hline No & System & $m_{0}$ & $n$ & $E$ & $E_{\mathrm{d}}$ & $E_{1}$ \\
\hline 1 & $\mathrm{Nb}+\mathrm{B}$ & 23 & 0 & 50 & 58 & 23 \\
2 & $\mathrm{Nb}+2 \mathrm{~B}$ & 12 & 0 & 35 & 58 & 15 \\
3 & $\mathrm{Ta}+2 \mathrm{~B}$ & 18 & 0 & 50 & 60 & 21 \\
4 & $\mathrm{Zr+2B}+308 \mathrm{ZB} 2$ & 10 & 0 & 34 & $34-42$ & 11 \\
5 & $\mathrm{H} f+2 \mathrm{~B}$ & 15 & 0 & 40 & 48 & 29 \\
6 & $\mathrm{Zr}+\mathrm{Si}$ & 8 & 0 & 30 & 56 & 20 \\
7 & $\mathrm{Ti}+\mathrm{Si}$ & 0 & 1 & 28 & 39.7 & \\
8 & $\mathrm{Ti}+\mathrm{C}$ \\
9 & $\mathrm{Ti}+\mathrm{O} .8 \mathrm{C}\}$ & 0 & 7 & 55 & & \\
10 & $\mathrm{Ti}+\mathrm{O} .5 \mathrm{C}$ & & & & &
\end{tabular}

Besides the experimentally established values $m_{0}, n$ and the value of activation energy $E$ Table 3 contains tabular values of activation energy $E_{d}$ of diffusion of non-metal $\mathrm{B}, \mathrm{Si}$ and $\mathrm{C}$ into the corresponding metal (ref. ${ }^{\mathrm{d}} 16$ ); Table 3 also shows the values of the activation energy of governing stage $E_{1}$ 
which can be derlved from the obtained profiles $T(x), \Phi(x)$ and $\eta(x)$ for each regime of synthesis by the equation (ref. 17):

$$
E_{l}=m_{0} R T_{m}^{2} /\left(T_{f}-T_{0}\right)
$$

Here: $T_{m}$ is the temperature of $\max \phi(x)$. Table 3 shows that in most cases the exponential kinetic law with the large parameter $m_{0}$ is active. This law is typical for the SHS waves of borides and some silicides (titanium silicide is an exclusion). Reaction deceleration can be accounted for by the enlargement of the product layer on the metal particles. Diffusion of non-metal through the product limits the process of product formation. The obtained values of $E$ could be expected to coincide with the tabural values $E_{\text {a }}$ of the corresponding metal and non-metal couples. However, as it is seen from Table 3 , the values of $E$ are less than that of $E_{f}$. This can probably be accounted for by the fact that $E_{d}$ generally corresponds to the volumetric diffusion while $E$ corresponds to surface diffusion which is due here to the fine-grained crystalline structure of metal at the beginning of the reaction zone (before the metal begins to melt). The values of $E_{\text {f }}$ are still smaller. It can be assumed that the small values of $E_{1}$ can be accounted for by the highly defective crystalline structure of the metal particles near $\mathrm{T}_{\mathrm{p}}$.

Thus in the system under consideration $M e+\alpha B$ and Me $+\alpha S i$ in the SHS waves the activation energy increases considerably as the reaction progress.

The mechanism of carbid formation in synthesis waves is different. The main difference lies in the fact that the propagation rate SHS waves and the process of synthesis at the beginning of the reaction are limited by the transfer of carbon to the titanium particle surface rather than by the process of diffusion of non-metal into metal through the layer of intermediate product. Soot generally used in the composition $\mathrm{Ti}+\alpha \mathrm{C}$ as a carbon containing substances has a complex structure and when it is submitted to the heat in the SHS wave it undergoes all kind of transformations accompanied by heat release. It is these transformations, leading to carbon reaching the surface of the particles of $\mathrm{Ti}$, that determine the wave rate. Indeed, the analysis of experiments reveals certain universal dependencies: the wave rate is completely determined by the type of soot (other parameters of the system being constant). The lower the stability of soot, the higher is the rate of synthesis wave propagation. In ref. 18 it was shown that nonstabjlity of soot can be described in terms of a set of $x$-ray structural parameters obtained from the $\mathrm{X}$-ray diffraction spectra of these soots. A combination of these parameters constituting the dimensionless complex $\mathrm{P}_{\mathrm{k}}$ that yields the correlation with the SHS wave propagation rate $\tau_{6}$ was found. The modified complex $P_{k}$ for systems $\mathrm{Ti}+\alpha \mathrm{C}$ and $\mathrm{Zr}+\alpha \mathrm{C}$ looks as follows (ref. 4):

$$
P_{k}=\rho_{0} / A\left(R_{0}^{-1} \cdot M \cdot \operatorname{ctg} \varphi_{0}\right)\left(L_{c} / L_{a} \cdot{ }^{d_{002}} / d_{0}\right)
$$

Here: $\rho_{0} / A$ - coefficient; factor $\operatorname{ctg} \varphi_{/} / R_{0}$ describes the disorientation of crystallite in soot particles; factor $M$ describes the degree of homogeneity of crystallite in soot particles; doo2/ $d_{0}$ describes the increase of relative distance between flat carbon lattices of crystallite ( $d_{0}$ an absolute distance between flat carbon lattices of graphite); Lc/La describes the relative part of crystalite surface with available bonds.

The higher each of these factors, the higher is the nonstability of soot. Dependencies of propagation rate $\tau_{B}$ of mixtures $T i+\alpha C$ and $Z r+\alpha C$ on the modified complex $P_{\alpha}$ are shown in Fig. 7 . It can be seen that universal dependencies $\tau_{b}\left(P_{x}\right)$ can be obtained for each metal and each value $\alpha$.

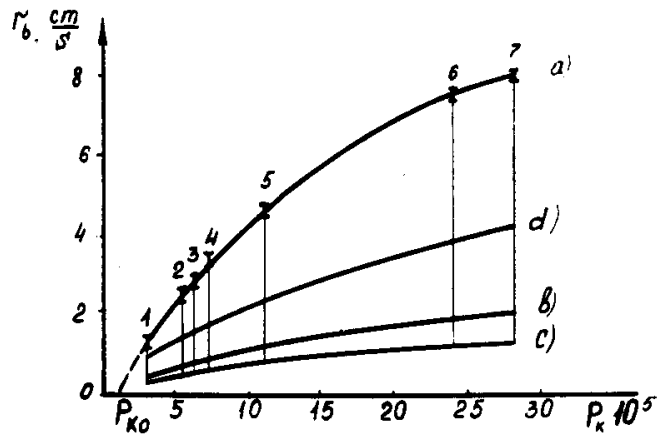

$$
\begin{aligned}
& \text { Fig. } 7 \text {. Dependencies } \\
& \text { of propagation rate } z_{6} \\
& \text { on complex } P_{\kappa} \\
& \text { System } \mathrm{Ti}+\alpha \mathrm{C}: \\
& \text { a) } \alpha=1.0 ; \\
& \text { b) } \alpha=0.6 ; \\
& \text { c) } \alpha=0.5 ; \\
& \text { System } \mathrm{Zr}+0.6 \mathrm{C} \rightarrow \mathrm{d}) \\
& \text { Types of soot: } \\
& \text { 1. PM-75;2.DG-100; } \\
& \text { 3. PM-105; 4.PM-50; } \\
& \text { 5. PGM-33; 6.PM-15; } \\
& \text { 7. TG-10. }
\end{aligned}
$$


Thus, Fig. 7 proves that the transformation of soot in SHS wave and the transfer of carbon to the surface of metal particles during the process of this transformation limit the propagation rate of carbide synthesis waves.

\section{REFERENCES}

1. A.G. Merzhanov, In book: Physical Chemistry. Modern problems. Ed. by Ja.M. Kolotirkin, Moscow, "Chemistry" (1983), (in Russian).

2. A.A. Zenin, Doct. Dissertation, Inst. of Chem. Phys. USSR Acad.of Sci., Moscow (1976) (In Russian).

3. G.A. Nersisjan, Dissertation, Inst, of Chem. Phys, USSR Acad. of Sci., Moscow (1980) (in Russian).

4. Yu.V. Turkin, Dissertation. Inst. of Chem. Phys. USSR Acad. of Sci., Moscow, (1986) (in Russian).

5. A.P. Aldushin, A.G. Merzhanov, B.I. Khaikin, Compt.Rend. Acad. Sci. USSR, 204, No.5; 1139-1142 (1969). (in Russian).

6. A.A. zenin, A.G. Merzhanov, G.A. Nersisjan, Compt. Rend. Acad.Sci.USSR, 250, No.4, 880-884 (1980) (in Russian).

7. A.A. Zenin, G.A. Nersisjan, Thermal Structure of sHs Wave, Mechanism and Macrokinetics of High-Temperature Non-Isothermal Interaction of Elements in the System Ti-Si and Zr-Si. Preprint Chernogolovka (1980) (in Russian).

8. A.A. Zenin, in book: Physical Processes with Combustion and Explosion, 68-104, Atom Publishing House, Moscow (1980) (in Russian).

9. A.G. Merzhanov, E.N. Rumanov, B.I. Khaikin, Journ. Appl.Mech. and Techn. Phys., USSR, No.6, 99-105 (1972) (in Russian).

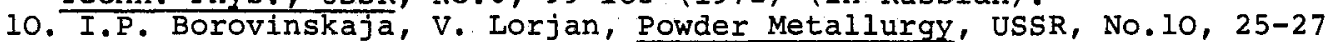
(1973) (in Russian).

11. Z.A. Munir, 3rd Intern. Sem. on Flame Structure, Reports, Alma-Ata (1989).

12. A.A. Zenin, A.G. Merzhanov, G.A.Nersisjan, The Investigation of Thermal Wave Structure in SHS Processes on Example Boride Synthesis. Preprint, Chernogolovka (1980) (in Russian).

13. W.A. Petrov, I.A. Weshnevetskaja, A.N. Winnikov, Thermo- and Electroconductivity of Carbide of Transient Metals of the Groups 4 and 5 . Moscow "Standards" (1982) (in Russian).

14. S.W. Shulepov. Physics of carbon-graphite materials. Cheljabinsk (1968) (in Russian).

15. B.I.Khaikin, In book: Combustion processes in the Chemical Technology and Metallurgy, 227-244 (1975) (in Russian).

16. G.B. Samsonov, I.M. Vinitsky, Refractory Compositions. Reference Book. Moscow, "Metallurgy" (1976) (in Russian).

17. A.P. Aldushin, T.M. Martemjanova, A.G. Merzhanov, B..I. Khaikin, K.G. Shkadinskij. The Phys. of Comb. and Expl., USSR, No.5, 613-626 (1973) (in. Russian).

18. A.A. Zenin, Yu.M. Korolev, V.T. Popov, Yu.V.Turkin, Compt. Rend. Acad. SCi. USSR, 287 , No.1, $111 \rightarrow 13(1986)$ (in Russian). 